\title{
Multifractal characteristics of magnetospheric dynamics and their relationship with sunspot cycle
}

\author{
Sumesh Gopinath and P.R. Prince* \\ Department of Physics, University College, Trivandrum-695034, Kerala, India
}

\begin{abstract}
Multifractal analysis deals with a process whose power-law scaling behavior is a nonlinear function of statistical moments having a spectrum of scaling exponents. In contrast, monofractal process has a scaling behavior which is a linear function of moments with a single scaling exponent. In this study, multifractal analysis of complex magnetosphere using boxcounting approach has been considered for a better understanding of intermittent and persistent features, focusing on the auroral electrojet index (AE), SYM-H and Dst indices. For the analysis, 1-min AE, SYM-H and Dst indices are taken during the interval 1985-2007. We compare the sunspot cycle dependence of self-similarity and multifractality of magnetospheric proxies such as AE, SYM-H and Dst indices, using both monofractal and multifractal paradigms. The results indicate that monofractal features of AE, SYM-H and Dst indices are solar activity dependent. But, while analyzing the multifractal features, multifractal spectrum of AE index is less dependent on solar activity when compared with that of SYM-H and Dst indices. This implies that, other than solar wind forcing, certain complex phenomena of internal origin also modify the dynamics of geomagnetic fluctuations in the high-latitude auroral region.
\end{abstract}

Keywords: Multifractality; Magnetosphere; Sunspot cycle

*Corresponding author. Tel. +9495211211

E-mail address: princerprasad@ gmail.com 


\section{Introduction}

The Earth's magnetosphere often reflects complex spatio-temporal dynamics where power-law scaling associated with intermittency has been considered as the hallmark of a turbulent system. Variations in the scaling behavior of magnetospheric proxies, such as auroral indices, represent typical signatures of cooperative dynamics and non-Markovian features. These also reflect the presence of underlying long-range interactions which may develop, even internally without an external driver, in the magnetosphere. Magnetospheric activity during substorms is a multiscale process which extends from small-scale processes such as magnetohydrodynamic (MHD) turbulence and current disruption to large-scale processes like plasmoid ejection and global convection.

The non-equilibrium phenomena in the magnetosphere have a foothold on the theories that the magnetospheric dynamics exhibits global coherence as well as low dimensional behavior [Vassiliadis et al., 1990; Sharma, 1993, 1995; Sharma et al., 1993; Sitnov et al., 2000]. Meanwhile, the turbulent aspects of the magnetosphere are intimately related with fractal structure where the plasma turbulence in the distant magnetotail possesses self-organization characteristics which have been described in terms of the fractal properties [Zelenyi et al., 1998]. Dynamical systems in the state of self-organized criticality (SOC) are considered as driven farfrom-equilibrium which produce self-similar distributions of individual disruption events, called as avalanches [Bak et al., 1987]. The role of the SOC component in the intermittent dynamics of magnetosphere, considering its most probable location to be in the plasma sheet has also been a topic of extensive research for the past few years [Bak et al., 1988; Angelopoulos et al., 1994; Consolini et al., 1996; Uritsky and Pudovkin, 1998; Lui et al., 2000]. 
Numerous studies have considered fractal nature of interplanetary magnetic field, geomagnetic indices such as auroral electrojet (AE) index and geomagnetic field components, which is still a promising way of analyzing the turbulence which exists in solar windmagnetosphere-ionosphere system [Mandelbrot, 1985; Burlaga, 1986; Uritsky and Pudovkin, 1998; Chang, 1999; Vörös, 2000; Wanliss, 2005; Dobias and Wanliss, 2009; Consolini, 2011; Macek, 2007; Bolzan and Rosa, 2012]. Monofractals are invariably self-similar or scale invariant where as multifractal processes extend the notion of similarity to generalized scaling (or multiscaling) that includes both long-memory as well as extreme variations. A self-similar exponent (or Hurst exponent) physically quantifies the magnitude of the effect of excitation associated with the preceding event on succeeding events or the degree of persistence/antipersistence observed in the dynamics [Bunde and Havlin, 1994; Consolini and Lui, 1999].

Geomagnetic storm is termed as a major disturbance in the magnetospheric activity that may vary from certain hours to a few days in which solar wind energy is coupled to the magnetosphere-ionosphere system. This leads to intensification in ring current (RC) which is sufficient to exceed a threshold of disturbance quantifier called as the Dst index [Gonzalez et al., 1994]. Auroral electrojet (AE) index, which quantifies the global electrojet activity, with a resolution of 1-min are derived from the H-component of geomagnetic fluctuations measured at auroral-region observatories [Davis and Sugiura, 1966]. The SYM-H index has been used as a descriptor of symmetric disturbance fields in the horizontal direction at midlatitudes. Both SYM$\mathrm{H}$ and Dst quantifies the horizontal geomagnetic field intensity based on a coordinate system and set of stations that differ slightly, although both are considered as descriptors of magnetospheric disturbances [Sugiura and Poros, 1971]. 
The multifractal box-counting method is acclaimed as the most popular method for determining the intermittent features of large multifractal time series data. It can be considered as a much simpler and faster method for measuring the multifractality of stochastic data sets and many works have been reported recently that employs the modified box-counting technique [Roy et al., 2007; de Souza and Rostirolla, 2011; Domínguez et al., 2014]. During the past three decades, several box-counting algorithms have been proposed for computing fractal properties in order to improve the accuracy of estimation and to decrease the computation time [Hou et al., 1990; Kruger, 1996]. The method relies on the fact that by plotting a time series and consider it to be a curve on the plane, we can cover it with equal size boxes; counting the number of points that fall within each box leads to the fractal dimension of the series [Addison, 1997].

Our major aim in this paper is to compare the variations in solar activity dependence of magnetosphere system evaluating monofractal Hurst exponent (using rescaled range analysis) and multifractal measures such as singularity strength (using multifractal box-counting method), calculated from 1-min geomagnetic proxies such as AE, SYM-H and Dst during the period 19852007. We have also performed surrogate data analysis of disturbance and calm times by taking AE and SYM-H data during Bastille day event (July 13-19, 2000) as the disturbance phase and seven days prior to the event as the calm phase. This shows that the box-counting approach certainly could differentiate actual data from surrogates as well as storm times from calm times. The rest of the paper is structured as follows. The data used for the present work are discussed at first; then box-counting technique to evaluate multifractality is summarized briefly. After this, box-counting technique is applied to the aforementioned proxies and lastly, the implications of the results are discussed. 


\section{Data}

The AE index with 1-min resolution for a period of 1985-2007 has been obtained from the World Data Center for Geomagnetism, Kyoto, Japan. The 1-min SYM-H data for a period of 1985-2007 has been taken from the OMNI dataset. The 1-min USGS Dst index for a period of 1985-2007 has been taken from USGS database. For the comparative analysis of solar activity during different solar cycles, the major solar activity proxy called sunspot number (SSN) has also been taken for the period of 1985-2007. The Solar Influences Data Analysis Center (SIDC) at Royal Observatory of Belgium provides the daily sunspot number (SSN) data.

\section{Methods}

\subsection{Multifractal analysis: The box-counting method}

Fractals are geometrical objects/functions whose subsets show fractal geometry and have a power-law dependence on the scale such that the exponent is the fractal dimension $D_{H}$. The fractal dimension is usually calculated by enclosing the object with hyper boxes of side, say $\mu \leq L$, and a hyper volume given by $\mu^{D_{f}}$ where $D_{f} \geq D_{H}$ is the dimension of the embedded Euclidean space. Now, if $N_{b}(\mu)$ denotes the number of boxes that contain points $N_{b}(\mu) \sim \mu^{-D_{f}}$, then $D_{f}$ can be written as

$D_{f}=\lim _{\mu \rightarrow \infty} \frac{\log N_{b}(\mu)}{\log \mu}$

If $P_{i}$ are the normalized probabilities given as $N_{i}(\mu \leq L) / N$ (for measure in the $i^{t h}$ box, in the resolution $\mu$ ), where $\mathrm{N}$ is the total number of points and $N_{i}$ is the number of points in the $i^{t h}$ box then for a multifractal measure $P_{i}(\mu)$ will scale as $\mu^{\alpha_{i}}$ where $\alpha_{i}$ are local Lipschitz-Hölder exponents that denote the singularity strength. 
Now, we can define a partition function such that

$Z_{q}(\mu)=\sum_{i}^{n_{i}(\mu)} P_{i}^{q}$

where $q$ is the moment order and $n_{i}(\mu)$ are the number of boxes in the scale $\mu$. If the distribution of the measure is multifractal, $Z_{q}(\mu)$ will also scale as $\mu^{\tau_{q}}$ where $\tau$ is the mass exponent of order $q$

$\tau_{q}=\lim _{\mu \rightarrow 0} \frac{\log z_{q}(\mu)}{\log \mu}$

Generally, to be an approximate multifractal, the relationship $\log Z_{q}$ against $\log \mu$ should be well described by a linear relationship, even though such a relationship with superimposed oscillations is also considered.

If $\alpha(q)=\frac{d \tau_{q}}{d q}$, the $f(\alpha)$ is the fractal dimension of the set of boxes with singularity strength $\alpha$. If we consider the two conjugate pairs $\{f(\alpha), \alpha\}$ and $\{\tau(q), q\}$, then the Legendre transform between them yields

$\tau(q)=q \alpha(q)-f(\alpha(q))$

In other words, $\alpha(q)$ is that value of the Lipschitz-Hölder exponent, which maximizes $[q \alpha-f(\alpha)]$ for a given $q$. Due to finite size effects, the box-counting algorithm may not evaluate points of $f(\alpha)$ curves for $q \rightarrow \pm \infty$, mainly for negative $q$ 's. It results in lacking of points which occurs mainly in the right part of the $f(\alpha)$ curve where the partition function is weighted with negative moments $(\mathrm{q}<0$ in Eqn. 3). In such cases, the whole multifractal spectrum may be estimated by interpolation. 
Also, the generalized q-order self-similar exponent $H_{q}$ can be expressed by the relation

$q H_{q}=\tau(q)+1$

According to theory, $\Delta \alpha=\alpha_{\max }-\alpha_{\min }$ should be zero for monofractals, but usually $f(\alpha)$ spectra for monofractals show a narrow $\Delta \alpha$ due to finite size effects. For more details of the modified box-counting method using Hou's algorithm the reader is referred to the article of de Souza and Rostirolla (2011).

\subsection{Rescaled Range (R/S) analysis}

The Hurst exponent, which characterizes the scaling behavior of the range of cumulative deviations of geomagnetic indices from its average, can be computed using the method called R/S analysis, which was proposed by Mandelbrot and Wallis (1969). Rescaled range characterizes the divergence of time series measured as a ratio of the range of the mean-centered values for a particular duration and standard deviation for that duration. The range $\mathrm{R}$ denotes the difference between the maximum and minimum accumulated values or the cumulative sum at discrete time, say $t$, over a particular time period $\tau$.

$R(\tau)=\max \{x(t, \tau)\}-\min \{x(t, \tau)\}$

and the standard deviation $S$ is given as

$S=\left\{\frac{1}{\tau} \sum_{t=1}^{\tau}\left[y\{t\}-\langle y\rangle_{\tau}\right]^{2}\right\}^{1 / 2}$

where $\langle y\rangle_{\tau}=\frac{1}{\tau} \sum_{t=1}^{\tau} y(t)$ and $x(t, \tau)=\sum_{n=1}^{t}\left[y(n)-\langle y\rangle_{\tau}\right]$.

Now, 
$R / S=(k \tau)^{H}$

where $H$ is the Hurst exponent and $k$ is a constant. The $R / S$ method can be considered as a direct monofractal method for the analysis of the solar activity variations of geomagnetic indices.

\subsection{Complete Ensemble Empirical mode decomposition with adaptive noise}

For identifying the oscillatory components or intrinsic cycles embedded in a stochastic time series, empirical mode decomposition can be successfully employed. It has the potential to even analyze nonlinear and non-stationary data [Huang et al., 1998; Huang and Wu, 2008]. The salient characteristic of the empirical mode decomposition (EMD) is that a stochastic time series could be easily decomposed into a number of constituent mono-components called modes or more precisely, intrinsic mode functions (IMFns). EMD will consider a mode as IMFn if only it satisfies two basic conditions:

(1) The modes should possess the same number of extrema and zero crossings or differ by one utmost.

(2) The maximal and minimal envelopes of the modes should be symmetric.

The fundamental notion of EMD is to extract all constituent structural components from a time series beginning from the one having the maximum frequency and ending with the one having minimum frequency. At first, by identifying maxima and minima, the intrinsic harmonics are locally extracted. Then, repeating this strategy for the entire time series, the component having the highest frequency can be determined. If it is detached from the actual series, a new series can be generated which contains several intrinsic components, but will have only those frequencies lower than the one already extracted. Finally, the actual time series has been 
decomposed into a number of cyclic modes and on most occasions, a residual trend indicated as $r_{m}$ in equation (9).

$x(i)=\sum_{k=1}^{m} I M F n_{k}(i)+r_{m}(i)$

where $x(i)$ is the time series which gets decomposed into $m$ IMFns, $I M F n_{k}, k=1 \ldots m$ and a residual trend $r_{m}$. In the present work, a modified EMD algorithm based on ensemble empirical mode decomposition (EEMD) called as complete ensemble empirical mode decomposition (CEEMD) which is a noise assisted empirical mode decomposition algorithm has been used. The CEEMD works by adding certain amplitude of white noise to a time series, decomposing it through EMD, and obtaining the result. In contrast to the ensemble empirical mode decomposition (EEMD) method, the CEEMD also ensures that the IMFn set is quasi-complete, noise-free as well as orthogonal. The CEEMD has the potential to ameliorate mode mixing and intermittency problems. According to the CEEMD procedure, a positive and negative white noise $\eta^{+}(t)$ and $\eta^{-}(t)$ is added to the geomagnetic time series $x(t)$ to get $y^{+}(t)$ and $y^{-}(t)$ such that,

$$
\begin{aligned}
& y^{+}(t)=x(t)+\eta^{+}(t) \\
& y^{-}(t)=x(t)+\eta^{-}(\mathrm{t})
\end{aligned}
$$

Then, each of the new data $y^{+}(t)$ and $y^{-}(t)$ are decomposed using the EMD method and two sets of IMFns for the $y^{+}(t)$ and $y^{-}(t)$ are obtained. Now, it is possible to obtain the decomposed result by averaging the $I M F n_{k}(i)$ where $I M F n_{k}(i)$ represents the $i^{\text {th }}$ IMFn of the $k^{\text {th }}$ iteration. Since CEEMD is computationally expensive and take significant amount of time for computations, an improved version called as complete ensemble empirical mode 
decomposition with adaptive noise (CEEMDAN) has been proposed [Torres et al., 2011]. For more details of the CEEMDAN method and algorithm the reader is referred to the article of Torres et al., (2011). For the present analysis, number of ensemble trials is set as 100. As suggested by Wu and Huang (2009), the amplitude of the added white noise was set as 0.2 times the standard deviation of the actual series and has been verified that there is neither the presence of mode-mixing nor redundant IMF components exist.

\section{Results and Discussion}

\subsection{Fractal analysis of AE and SYM-H indices: Features during storm and calm periods}

In the present study, we have proposed box-counting methods for the identification of intermittency of magnetospheric proxies, which display multifractal features of magnetosphere during disturbance as well as calm times. For the multifractal quantification of storm and calm times, we mainly use the multifractal parameters such as singularity strength $\left(\Delta \alpha=\alpha_{\max }-\right.$ $\left.\alpha_{\text {min }}\right)$ and degree of persistency $\left(\Delta H=H_{\max }-H_{\min }\right)$. For sliding window analysis of sunspot cycle dependence of multifractality we use only the singularity strength $\Delta \alpha$ of geomagnetic proxies. Even though we have not considered $\Delta H$ for sliding window analysis of sunspot cycle dependence, we are confident that the variations of $\Delta H$ will also yield similar variations like $\Delta \alpha$. For monofractal quantification of storm and calm times as well as sliding window analysis of sunspot cycle dependence of monofractality, we use only the Hurst exponent computed with R/S analysis.

Figure 1 shows the variations of SYM-H index and AE index during the Bastille day event July 13-19, 2000 (marked as the storm phase) which is an extreme geomagnetic storm event and 7 days prior to the event from July 06-12, 2000 (marked as the calm phase). It is to be 
noted that the calm phase can be considered 'quiet' only in comparison with the geomagnetic storm event, especially in the case of $\mathrm{AE}$ index. For calculating the calm and storm time variations, we consider it as the most appropriate method since it is extremely difficult to obtain prolonged duration of true quiet times of AE index even in the absence of geomagnetic storms. The surrogate data analysis includes the creation of an ensemble of data sets using Monte Carlo methods which are consistent to a null hypothesis [Theiler et al., 1992]. It has been accepted that the surrogate analysis could be a promising tool for the discrimination of multifractality present in an experimental data. The typical intermittent features of the AE index are compared with those of linear time series or surrogates, which have the same autocorrelation function and the power spectrum of the actual time series. In the present study, we used the improved algorithm of Schreiber and Schmitz (1996) to create an ensemble of 40 surrogates. Beginning with a white noise signal, its Fourier amplitudes are replaced by the corresponding amplitudes of the original data. The rank-order of the derived data is used to reorder the actual time series. The process is repeated multiple times until the change in the matching of power spectrum is negligible.

Figure 2 shows the surrogate data analysis of multifractal parameters for AE index considering the Bastille day geomagnetic storm event and the comparative calm time prior to the event (as shown in Fig. 1(b)). Figure 2(a)-(c) respectively shows the variations of multifractal features during disturbance periods such as the variations of mass exponents $\tau(q)$ against $q$, variations of $f(\alpha)$ versus singularity strength $\alpha$ and variations of generalized Hurst exponent $H(q)$ against $q$. Likewise, figure 2(d)-(f) respectively shows the variations of multifractal features during quiescent periods such as the variations of mass exponents with $q$, variations of $f(\alpha)$ versus $\alpha$ and variations of generalized Hurst exponent $H(q)$ versus $q$. The bold magenta (for storm time) and cyan (for calm time) colored curves represent multifractal parameter of the 
actual index while mean values of parameters for surrogates are represented as bold blue curves. The corresponding sum and difference of the mean value and the standard deviation is represented as dotted blue curves. Similarly, figure 3(a)-(c) respectively shows the variations of multifractal features during disturbance periods and figure 3(d)-(f) respectively shows the variations of multifractal features during calm periods. The mass exponent spectra, $f(\alpha)$-spectra and generalized Hurst spectra of both geomagnetic indices certainly show drastic variations, with respect to surrogates, during both calm and storm phases. Thus the surrogate analysis clearly shows that the nature of multifractality of AE and SYM-H index is quite different from that of the surrogate data. From the Figs. 2 and 3, it is also certain that box-counting technique could capture varying degrees of complexity of magnetosphere and could quantify the multifractality of AE and SYM-H indices.

We can compare the singularity strength from $f(\alpha)$-spectra of actual storm and calm times of AE index using a moving window of 3 days with 1 hour separation between adjacent windows. The choice of the window width of 3 days with a separation width of 1 hour is suitable to analyze an event having 7 days duration as it ensures both sufficient time series length as well as adequate $\Delta \alpha$ points to calculate the average value and standard errors. Here we can see that the average $\Delta \alpha_{\text {storm }}=1.7849 \pm 0.0171$ while $\Delta \alpha_{\text {calm }}=1.6989 \pm 0.0142$. The location and the spread of the auroral electrojet currents are subject to rapid temporal variations depending on the levels of geomagnetic activity. While both the diameter of the polar cap and width of the polar oval expand during active conditions, they contract relatively to a narrow region around the magnetic pole during quiescent times. Multifractal spectrum shows evidences of dynamical phase transition of the magnetosphere system as the system is continuously driven between quiet and disturbed periods. 
Similarly, if we compare the singularity strength of multifractal spectra of actual storm and calm times of SYM-H indices, we can see that $\Delta \alpha_{\text {storm }}=2.0504 \pm 0.0244$ while $\Delta \alpha_{\text {calm }}=1.8391 \pm 0.0073$. The singularity strength $\Delta \alpha$ of calm times are less than the storm periods, which certainly shows that during self-organized disturbed periods long-range correlations spontaneously emerge in the dynamical behavior of the system when compared with decorrelated quiescent times. During severe magnetospheric storms, the auroral electrojet system shifts drastically towards the equator. The midlatitude current system will be affected by these transitions which in turn show its signatures on SYM-H index. The multifractal analysis thus invariably reflects intermittent nature of AE and SYM-H indices as the fluctuation changes from regularity/synchrony to irregularity/asynchrony or vice versa. If we compare the degree of persistency of storm and calm times of geomagnetic indices, the $\Delta H$ has been seen increased during storm times when compared with quiet times. For the $\mathrm{AE}$ index, $\Delta H_{\text {storm }}=1.4739 \pm$ 0.0173 while $\Delta H_{\text {calm }}=1.4085 \pm 0.0145$. For the SYM-H index, $\Delta H_{\text {storm }}=1.609 \pm$ 0.0241 while $\Delta H_{\text {calm }}=1.4877 \pm 0.0071$. Our method also goes well with the earlier works of Consolini et al. (1996), Uritsky and Pudovkin (1998), Rypdal and Rypdal (2010) and Pavlos et al. (2012) who gave evidence suggesting that the behavior of some geomagnetic indices (like auroral electrojet indices) and quantities associated with the solar wind (like certain plasma bulk parameters) cannot be constrained as only self-similar. They are inherently multifractal as well and those descriptions which mark them as monofractal are valid only to a limited extent.

Figure 4 shows the R/S analysis of 1-min AE and SYM-H indices taken during the geomagnetic storm and calm periods. The data used for the analysis is the Bastille day event and the calm phase prior to the disturbance time as shown in Fig. 1. The smallest box size the sample can be divided into is taken as 50 . The number of divisors used for the $\mathrm{R} / \mathrm{S}$ analysis is 48 . Even 
though there are different scaling regions, linear fit has been employed to get the mean exponent value. Price and Newman (2001) reported the R/S analysis of AE index, which can also be considered as a powerful method to determine self-similarity in a monofractal context. For the AE index, the Hurst exponent during storm time is obtained as 0.9171 while during calm time it gets decreased to 0.8896. Similarly, for the SYM-H index, the Hurst exponent during storm time is found to be 0.9865 and gets decreased during calm time to 0.942 . On the average, AE index is found to be having a lower persistency when compared with the SYM-H index.

Wanliss (2005), while studying Dst index with detrended fluctuation analysis (DFA), noted an increase in the associated scaling exponent during disturbance times, while the value of the scaling exponent nearly resembles that of a pure Brownian motion during calm times. While quantifying the persistency of $\mathrm{AE}$ and Dst indices near the onset of magnetospheric disturbances such as geomagnetic storms and substorms, an increase in the value of Hurst exponent has also been reported earlier [Uritsky and Pudovkin, 1998; Balasis et al., 2006]. These impulsions can be attributed to the increase in time correlation or long-term memory in the associated proxies related with the magnetospheric dynamics. In an out-of-equilibrium system such as the Earth's magnetosphere, the temporal scale-dependent transitions in associated fluctuations of geomagnetic time series can be taken as a signature that accompanies a dynamical phase change [Sitnov et al., 2000, 2001; Consolini et al., 2008].

As we know, the magnetosphere is a dynamically complex and spatially extended plasma system, continuously driven between in-and-out-of-equilibrium globally by the solar wind turbulence. Hence storm-related emergence of distant coherences in associated time series are in fact fingerprints of spontaneous descent in degrees of freedom of the magnetosphere. Similar studies based on the nonlinear dynamics of such extended systems are carried out in different 
areas of space physics where the theory of self-organized criticality (SOC) provides a bridge between intermittency, self-similarity and power-law scaling [Wanliss et al., 2005; Macek, 2007; Macek and Wawrzaszek, 2011; Aschwanden, 2011]. It can be considered that during magnetic disturbance periods associated with geomagnetic storms and subsequent substorms, magnetotail plasma sheet has been repetitively steered into an unstable critical state and later back to stability characterized by robust self-organization, spatio-temporal complexity and scale-invariant processes. Such switching of states is found to develop naturally in space-plasmas with multiscale dissipative and energization processes, through spatio-temporal dynamics [Chang, 1992; Chang, 1999; Klimas et al., 2000; Valdivia, 2012]. Using rank-ordered multifractal analysis (ROMA), Consolini and De Michelis (2011) reconfirmed that AE index is a descriptor of two entirely different processes; the directly-driven convection by the energetic solar wind (featured by gradual increase of the electrojet intensity with time) followed by an impulsive unloading (rapid increase of AE index).

\subsection{Fractal analysis of AE, SYM-H and Dst: Sliding-window based sunspot cycle dependence of $\Delta \alpha$ and $H_{R / S}$}

The temporal variations of multifractal spectrum of AE index during the period of 19902007 and SYM-H index during the period of 1985-2007 has been shown in figure 5(a) and 5(b) respectively. The values are computed for a moving window which is having a width of 30 days where adjacent windows are separated by a time span of 1 week. As we closely examine the figure 5(a), we cannot see any evidence of distinct solar maxima or minima from the AE spectra. But figure 5(b) clearly shows the presence of solar maxima and minima in accordance with the 11-year sunspot cycle. These points to the fact that the multifractality of AE index is independent of sunspot cycle while multifractality of SYM-H index is sunspot cycle dependent. 
For further analysis, we consider the sunspot cycle dependence of multifractal singularity strength $\Delta \alpha$ for AE, SYM-H and Dst indices using a sliding-window method, followed by the CEEMD analysis of $\Delta \alpha$. From the CEEMD analysis, the IMFn of $\Delta \alpha$ which reflect the 11-year periodicity of multifractality inherent in geomagnetic indices can be obtained. Usually, from the whole set of IMFns, where the characteristic scale increases with the mode number, the mode having the largest mode number is considered as the sunspot cycle variation. It is to be noted that the residue is not considered as an intrinsic mode. In other words, it will be the slowest varying mode having the maximum characteristic scale. In a 22 year data (1985-2007), harmonics lower than 11-year component usually does not appear as an intrinsic mode in EMD analysis. Even if present, such lower harmonics are eliminated along with the residue. For proper sunspot cycle dependence, the variations in solar activity proxies (such as sunspot number) corresponding to solar maxima and minima have to coincide with the variation of sunspot cycle mode of $\Delta \alpha$ of geomagnetic indices. The existence of slight time lags/leads can be attributed to the difference in geomagnetic activity during the ascending and descending phase of solar activity as reflected by the geomagnetic index [Ahn et al., 2000]. The data gaps in AE index during 1989-1990 have been filled using linear interpolation method.

Figure 6 shows the $\Delta \alpha_{A E}$ computed for different window lengths and sunspot cycle mode of its IMFn, evaluated during the period of 1985-2007. The $\Delta \alpha$ has been computed using 15-day, 30-day and 60-day windows in order to verify the stability of the result. For close evaluation, for the 15-day window, the adjacent windows are separated by a span as short as 1 day. For 30-day and 60-day windows, 1 day separation of adjacent windows will not produce any notable variation and hence 1 week separation has been used. The window span less than 15 days may generate erroneous results as our purpose is to study long-term variations rather than short-term 
fluctuations such as those caused by geomagnetic storms, flares and coronal mass ejections (CMEs) which may last for few hours to several days. Increasing the window widths from 60days will also be impractical as the number of data points will be significantly reduced which in turn affect the performance of EMD analysis. Similar to the procedure shown in Fig. 6, we can also compute the sunspot cycle modes of SYM-H and Dst indices. Figure 7 shows the sunspot cycle dependence of $\Delta \alpha$ of AE, SYM-H and Dst indices by considering sunspot cycle mode of $\Delta \alpha$ of corresponding indices on 15-day, 30-day and 60-day windows. The sunspot cycle mode of SSN is also given for comparison. The figure 7(a) shows that $\Delta \alpha_{A E}$ is independent of sunspot cycle while figure 7(b) and 7(c) shows that $\Delta \alpha_{S Y M-H}$ and $\alpha_{D s t}$ are sunspot cycle dependent. The Pearson's correlation coefficients of sunspot cycle mode of SSN and sunspot cycle mode of singularity strength of geomagnetic indices during the period of 1985-2007 are given in table 1. From the table it is seen that $\Delta \alpha_{A E}$ is poorly anti-correlated with sunspot cycle mode of SSN while $\Delta \alpha_{S Y M-H}$ and $\Delta \alpha_{D s t}$ are having high positive correlation. It is also seen that $\Delta \alpha_{D s t}$ is better correlated than $\Delta \alpha_{S Y M-H}$ against the sunspot cycle mode of SSN.

It is evident now that multifractality of SYM-H and Dst indices are certainly solar activity dependent while multifractality of $\mathrm{AE}$ index is independent. Although degree of multifractality is varying as a function of time, the observed intermittent behavior is more dependent on intrinsic dynamics of auroral-oval rather than on external forces related with solar activity. Such a behavior can be attributed to complex internal processes such as particle drifts, plasma convections and current flows at the altitudes related with the auroral activity and not fully of external magnetospheric origin as in the case of SYM-H or Dst indices where external ring current energization and dissipation play a dominant role in the development of multifractal features. The variations in IMFns of Dst and SYM-H indices can be attributed to the incomplete 
removal of solar-quiet trend from SYM-H index and also to the strong influence of certain highlatitude observatories which are highly influence by field-aligned currents (FACs) in deriving SYM-H index [Gannon and Love, 2011].

The evolution of Hurst exponent, $H_{R / S}$ computed using the rescaled range analysis is considered for the analysis of solar activity variations of self-similarity of geomagnetic indices in a monofractal paradigm. Figure 8 shows the sunspot cycle dependence of Hurst exponent $H_{R / S}$ of AE, SYM-H and Dst indices by considering sunspot cycle mode of $H_{R / S}$ of corresponding geomagnetic indices on 15-day, 30-day and 60-day windows. Here also the sunspot cycle mode of SSN is given for comparison. From the figure 8, it is evident that the self-similarity of AE, SYM-H and Dst indices are dependent on solar activity. The Pearson's correlation coefficients of sunspot cycle mode of SSN and sunspot cycle mode of $H_{R / S}$ of geomagnetic indices calculated for different windows, during the period of 1985-2007, are given in table 2. From the table it is seen that $H_{R / S}$ of AE, SYM-H and Dst are having high positive correlation. It is also interesting to see that on the average $H_{R / S}$ of Dst is having high correlation than $H_{R / S}$ of SYM-H. Likewise, on the average, $H_{R / S}$ of SYM-H is having high correlation than $H_{R / S}$ of AE. Hence it is certain that the behavior of some geomagnetic indices such as auroral electrojet indices cannot be constrained as only self-similar but they are essentially multifractal as well. The descriptions which mark them as monofractal are valid only to some extent as they cannot fully describe certain peculiarities of underlying dynamics regarding the intermittent behaviour of magnetosphere. Bursty behavior of the AE index is a combined effect of a multitude of localized events as well as inherent stochasticity of the system [Burlaga, 1995; Pulkkinen et al., 2006]. 
As we know, the auroral-oval widens equatorward during major disturbance times and shrinks poleward during very low activity periods, both outside the reach of auroral magnetic observatories. But they could still track certain phenomena which are limited mainly to the highlatitude auroral zones for most of the time such as energetic particle injections in the auroral zone, geomagnetic pulsations, auroral arcs and fluctuations related with auroral current system (field aligned currents and auroral ionospheric currents) that result from the interaction of solar wind-magnetosphere-ionosphere (SW-M-I) system. They could modulate the stochasticity as well as create typical intermittent structures in the complex dynamics of AE index. The most salient feature in the dynamics of AE index is the combined effects of geomagnetic storms and subsequent substorms. This produces some peculiar correlations in the magnetosphere which subsequently get reflected in the associated proxies, namely, auroral electrojets [Huang et al., 2003]. The AE index will also have signatures of isolated substorms other than storm-triggered ones, which are characterized by a group of phenomena during which varying degrees of spatiotemporal complexity in the magnetotail dynamics often take place [Consolini and Chang, 2001; Consolini, 2002]. The stochasticity of auroral indices could also be due to the fingerprint of oscillations such as the movement of magnetospheric cusps backward/forward, thus regulating the particle injections, during the times of maximum/minimum bulk plasma pressure of solar wind [Lundin et al., 2001; Balan et al., 2006]. Magnetospheric cusp turbulence was recently analyzed by Chang et al. (2010) using the ROMA method. They reported that ROMA spectrum tends to be smooth when there is fully developed fluid turbulence while unstable/undeveloped turbulence produces fluctuating spectrum. Intermittent features generated by intrinsic dynamics has also been reported in bulk plasma velocity and Alfvénic fluctuations of solar wind streams [Macek and Redaelli, 2000; Macek, 2007]. According to Consolini and Chang (2001) and 
references therein, turbulence has to be given more prominence than simple low dimensional chaotic nature, as indicated by the multifractality of $\mathrm{AE}$ index, to help in the studies of magnetospheric substorms. Hence it is also important to give attention to the intermittent turbulent features of the energy release fluctuations during substorms.

\section{Conclusion}

In the present study, we have used box-counting method for the identification of intermittency of magnetospheric proxies, which display multifractal features of magnetosphere during disturbance as well as calm times, focusing mainly on geomagnetic indices such as $\mathrm{AE}$ and SYM-H. Our study helps in quantifying the variations of dynamical patterns and associated scaling features with the magnitude of perturbations inherent in auroral electrojets. We have compared the solar activity dependence of self-similarity of AE, SYM-H and Dst indices using monofractal R/S method and multifractal box-counting approach. It is certain from our study that there exists solar activity dependence of self-similarity for AE, SYM-H and Dst indices in the monofractal case. But it is evident that, while the multifractality of Dst and SYM-H indices are dependent on solar activity, no such relationship can be seen with the multifractality of AE index. This ascertains the fact that multifractal approach is indeed necessary for the studies of intermittency in geomagnetic indices and for the further analysis of associated complex magnetospheric fractal dynamics. It is essential to emphasize that spatio-temporal dynamical complexity requires not only the occurrence of nonlinearities but also the intertwining of synchrony/regularity and asynchrony/irregularity. Our findings increase the possibility that understanding the fluctuations of multifractal features of geomagnetic indices associated with long-term solar activity have the potential for geomagnetic storm/substorm analysis. If the plasma sheet is considered to be residing in a self-organized state, then investigations on features 
related with different aspects of this peculiar state which are consistent with the intermittent behavior can be considered as a foot step towards a better understanding of the substorm evolution.

\section{Acknowledgements}

The anonymous reviewers are thanked for their meticulous reviews, which have helped improve the quality of the manuscript. The authors are thankful to World Data Center for Geomagnetism, Kyoto, Japan for providing auroral index data and hourly Dst index data (http://wdc.kugi.kyotou.ac.jp/) and to USGS for providing 1-min Dst index data (http://geomag.usgs.gov/). The authors thank OMNIWeb for providing 1-min SYM-H index and AE index for the period of 19812011(http://omniweb.gsfc.nasa.gov/), SIDC for providing sunspot numbers (http://www.sidc.be/silso/datafiles). One of the authors SG acknowledges a Junior Research Fellowship (Ac.EVI(4)/017465/2016) from the University of Kerala, Trivandrum. 


\section{References}

[1] Addison P. S. (1997). Fractals and Chaos: An Illustrated Course. Bristol and Philadelphia: Institute of Physics Publishing, London, United Kingdom.

[2] Ahn, B.-H., Kroehl, H. W., Kamide, Y. and Kihn, E. A. 2000b. Seasonal and solar cycle variations of the auroral electrojet indices. J. Geophys. Res. 62(14), 1301-1310, doi:10.1016/S1364-6826(00)00073-0.

[3] Angelopoulos, V., Kennel, C. F., Coroniti, F. V., Pellat, R., Kivelson, M. G., Walker, R. J., Russell, C. T., Baumjohann, W., Feldman, W. C. and Gosling, J. T. 1994. Statistical characteristics of bursty bulk flow events. J. Geophys. Res. 99, 21257-21280.

[4] Aschwanden, M. 2011. Self-Organized Criticality in Astrophysics: The Statistics of Nonlinear Processes in the Universe. Berlin: Springer

[5] Bak, P., Tang, C. and Wiesenfeld, K. 1987. Self-organized criticality: An explanation of 1/f noise. Phys. Rev. Lett. 59, 381-384.

[6] Bak, P., Tang, C. and Wiesenfeld, K. 1988. Self-organized criticality. Phys. Rev. A 38, 364374.

[7] Balan, N., Alleyne, H., Walker, S., Réme, H., Décréau, P. M. E., Balogh, A., André, M., Fazakerley, A. N., Cornilleau-Wehrlin, N., Gurnett, D. and Fraenz, M. 2006. Cluster observations of a structured magnetospheric cusp, Ann. Geophys., 24, 1015-1027.

[8] Balasis, G., Daglis, I., Kapiris, P., Mandea, M., Vassiliadis, D. and Eftaxias, K. 2006. From pre-storm activity to magnetic storms: A transition described in terms of fractal dynamics. Ann. Geophys. 24, 3557-3567.

[9] Bolzan, M. J. A. and Rosa, R. R. 2012. Multifractal analysis of interplanetary magnetic field obtained during CME events. Ann. Geophys. 30, 1107-1112, doi:10.5194 /angeo-30-1107-2012. 
[10] Bunde, A., and Havlin, S. eds. (1994). Fractals in Science, Springer, Berlin.

[11] Burlaga, L. F., and Klein, L.W. 1986. Fractal structure of the interplanetary magnetic field. J. Geophys. Res. 91(A1), 347-350.

[12] Burlaga, L. F. 1995. Interplanetary Magnetohydrodynamics, Oxford Univ. Press, New York.

[13] Chang, T. 1992. Low-dimensional behavior and symmetry breaking of stochastic systems near criticality - can these effects be observed in space and in the laboratory? IEEE Trans. Plasma Sci. 20(6), 691-694.

[14] Chang, T. 1999. Self-organized criticality, multifractal spectra, sporadic localized reconnections and intermittent turbulence in magnetotail. Phys. Plasmas. 6, 4137-4145.

[15] Consolini, G., Marcucci, M. F. and Candidi, M. 1996. Multifractal structure of auroral electrojet data. Phys. Rev. Lett. 76, 4082-4085

[16] Chang, T., Wu, C. C., Podesta, J., Echim, M., Lamy, H. and Tam, S. W. Y. 2010. ROMA (Rank-Ordered Multifractal Analyses) of intermittency in space plasmas - a brief tutorial review. Nonlin. Processes Geophys. 17, 545-551

[17] Consolini G. 2002. Self Organized Criticality: A new paradigm for the magnetotail dynamics. Fractals. 10, 275-283.

[18] Consolini, G., and Lui, A.T.Y. 1999. Sign-singularity analysis of current disruption. Geophys. Res. Lett. 26, 12, 1673-1676.

[19] Consolini, G., and Chang, T.S. 2001. Magnetic field topology and criticality in geotail dynamics: Relevance to substorm phenomena. Space Sci. Rev. 95, 309-321. 
[20] Consolini, G., De Michelis, P. and Tozzi, R. 2008. On the Earth's magnetospheric dynamics: Nonequilibrium evolution and the fluctuation theorem. J. Geophys. Res. 113: doi: 10.1029/2008JA013074.

[21] Consolini, G., and De Michelis, P. 2011. Rank ordering multifractal analysis of the auroral electrojet index. Nonlin. Processes Geophys. 18, 277-285.

[22] Davis, T. N. and Sugiura, M. 1966. Auroral electrojet activity index AE and its universal time variations. J. Geophys. Res. 71, 785-801 doi:10.1029/JZ071i003p00785.

[23] de Souza, J. and Rostirolla, S.P. 2011. A fast MATLAB program to estimate the multifractal spectrum of multidimensional data: Application to fractures. Comput. Geosci. 37, 2, 241-249, doi: 10.1016/j.cageo.2010.09.001

[24] Dobias, P., and Wanliss, J. A. 2009. Intermittency of storms and substorms: is it related to the critical behaviour? Ann. Geophys., 27, 2011-2018.

[25] Domínguez, M., Munoz, V. and Valdivia, J. A. 2014. Temporal evolution of fractality in the Earth's magnetosphere and the solar photosphere. J. Geophys. Res. Space Physics. 119, 3585-3603, doi:10.1002/2013JA019433.

[26] Ganon, J. L. and Love, J. J. 2011. USGS 1-min Dst index. J. Atm. Sol. Terr. Phys. 73, 323334.

[27] Gonzalez, W. D., Joselyn, J. A., Kamide, Y., Kroehl, H. W., Rostoker, G., Tsurutani, B. T. and Vasyliunas, V. M. 1994. What is a geomagnetic storm? J. Geophys. Res. 99, 5771-5792. [28] Hou, J., Gilmore, R., Mindlin, G.B., Solari, H.G. 1990. An efficient algorithm for fast $O(N \ln (N))$ box counting. Physics Letters A. 151, 43-46 doi:10.1016/0375-9601(90)90844-E 
[29] Huang, C.-S., Foster, J. C., Reeves, G. D., Le, G., Frey, H. U., Pollock, C. J. and Jahn, J.-M. 2003. Periodic magnetospheric substorms: Multiple space-based and ground-based instrumental observations. J. Geophys. Res. 108, 1411, doi:10.1029/2003JA009992.

[30] Huang, N. E., Shen, Z., Long, S. R., Wu, M. C., Shih, H. H., Zheng, Q., Yen, N.-C., Tung, C. C. and Liu, H. H. 1998. The empirical mode decomposition and the Hilbert spectrum for nonlinear and non-stationary time series analysis. Proc. R. Soc. London, Ser. A, 454, 903-993.

[31] Huang, N. E., and Wu, Z. 2008. A review on Hilbert-Huang transform: Method and its applications to geophysical studies. Rev. Geophys. 46, RG2006, doi:10.1029 /2007RG000228.

[32] Klimas, A. J., Valdivia, J. A., Vassiliadis, D., Baker, D. N., Hesse, M. and Takalo, J. 2000. Self-organized criticality in the substorm phenomenon and its relation to localized reconnection in the magnetospheric plasma sheet. J. Geophys. Res. 105(A8), 18,765-18,780.

[33] Kruger, A. 1996. Implementation of a fast box-counting algorithm. Computer Physics Communications. 98, 224-234. doi:10.1016/0010-4655(96)00080-X

[34] Lui, A. T. Y., Chapman, S. C., Liou, K., Newell, P. T., Meng, C. I., Brittnacher, M. and Parks, G. D. 2000. Is the dynamic magnetosphere an avalanching system? Geophys. Res. Lett. 27, 911-914.

[35] Lundin, R., Aparicio, B. and Yamauchi, M. 2001. On the solar wind flow control of the polar cusp. J. Geophys. Res. 106, 13,023-13,035.

[36] Macek, W. M. 2007. Multifractality and intermittency in the solar wind. Nonlin. Processes Geophys. 14, 695-700.

[37] Macek, W. M., and Redaelli, S. 2000. Estimation of the entropy of the solar wind flow. Phys. Rev. E. 62, 6496-6504. 
[38] Macek, W. M. and Wawrzaszak, A. 2011. Multifractal two-scale cantor set model for slow solar wind turbulence in the outer heliosphere during solar maximum. Nonlin. Processes Geophys. 18, 287-294.

[39] Mandelbrot, B. B. 1985. Self-affine fractals and fractal dimensions. Phys. Scripta. 32, 257260.

[40] Mandelbrot, B. B. and Wallis, J. R. 1969. Robustness of the rescaled range R/S in the measurement of noncyclic long-run statistical dependence. Water Resour. Res. 5, 967-988.

[41] Pavlos, G. P., Karakatsanis, L. P., Xenakis, M. N., Sarafopoulos, D. and Pavlos, E. G. 2012. Tsallis statistics and magnetospheric self-organization. Physica A. 391, 3069-3080.

[42] Price, C.P. and Newman, D. E. 2001. Using the R/S statistic to analyze AE data. J. Atm. Sol. Terr. Phys. 63, 1397-1397.

[43] Pulkkinen, A., Klimas, A., Vassiliadis, D. and Uritsky, V. 2006. Role of stochastic fluctuations in the magnetosphere-ionosphere system: A stochastic model for the AE index variations. J. Geophys. Res. 111, doi: 10.1029/2006JA011661.

[44] Roy, A., Perfect, E., Dunne, W.M. and McKay, L.D. 2007. Fractal characterization of fracture networks: an improved box-counting technique. J. of Geophys. Res. 112, B12201. doi:10.1029/2006JB004582.

[45] Rypdal, M. and Rypdal, K. 2010. Stochastic modeling of the AE index and its relation to fluctuations in $B_{z}$ of the IMF on substorm time scales. J. Geophys. Res. 115, A11216, pp 15. doi:10.1029/2010JA015463

[46] Schreiber, T., and Schmitz, A. 1996. Improved surrogate data for nonlinearity tests. Phys. Rev. Lett. 77, 635-638 
[47] Sharma, A. S., Vassiliadis, D. V., Papadopoulos, K. 1993. Reconstruction of lowdimensional magnetospheric dynamics by singular spectrum analysis. Geophys. Res. Letts. 20, $335-338$.

[48] Sharma, A. S. 1995. Assessing the magnetosphere's nonlinear behavior: Its dimension is low, its predictability, high. Rev. Geophys. 33, 645-650.

[49] Sitnov, M. I., Sharma, A. S., Papadopoulos, K., Valdivia, J. A., Klimas, A. J. and Baker, D. N. 2000. Phase transition-like behavior of the magnetosphere during substorms. J. Geophys. Res. $105,12955-12974$.

[50] Sitnov, M. I., Sharma, A. S., Papadopoulos, K., Vassiliadis, D., 2001. Modeling substorm dynamics of the magnetosphere: From self-organization and self-organized criticality to nonequilibrium phase transitions. Phys. Rev. E. 65, 016116.

[51] Sugiura, M., and Poros, D. J. 1971. Hourly values of equatorial Dst for the years 1957 to 1970. Rep. X-645-71-278, GSFC, Greenbelt, Maryland.

[52] Theiler, J., Eubank, S., Longtin, A., Galdrikian, B. and Farmer, J. D. 1992. Testing for nonlinearity in time series: the method of surrogate data. Physica D. 58, 77-94.

[53] Torres, M. E., Colominas, M. A., Schlotthauer, G. and Flandrin, P. 2011. A complete ensemble empirical mode decomposition with adaptive noise: ICASSP. IEEE. 4144-4147.

[54] Uritsky, V. M. and Pudovkin, M. I. 1998. Low frequency $1 / f$-like fluctuations of the AEindex as a possible manifestation of self-organized criticality in the magnetosphere. Ann. Geophys. 28, 1580-1588.

[55] Valdivia, J. A., Rogan, J., Muñoz, V., Toledo, B. A. and Stepanova, M. 2012. The magnetosphere as a complex system. Adv. Space Res. 51, 1934-1941. doi: 10.1016 /j.asr.2012.04.004 
[56] Vassiliadis, D. V., Sharma, A. S., Eastman, T. E., Papadopoulos, K. 1990. Low-dimensional chaos in magnetospheric activity from AE time series. Geophys. Res. Lett. 17, 1841-1844.

[57] Vörös, Z. 2000. On multifractality of high-latitude geomagnetic fluctuations. Ann. Geophys. $18,1273-1282$.

[58] Wanliss, J. 2005. Fractal properties of SYM-H during quiet and active times. J. Geophys. Res. 110, A03202, pp 12. doi:10.1029/2004JA010544

[59] Wanliss, J. A., Anh, V. V., Yu, Z. G. and Watson, S. 2005. Multifractal modeling of magnetic storms via symbolic dynamics analysis. J. Geophys. Res. 110, A08214, pp 11. doi:10.1029/2004JA010996

[60] Wu, Z. and Huang, N. E. 2009. Ensemble empirical mode decomposition: A noise-assisted data analysis method. Adv. Adapt. Data Anal.1, 1-41.

[61] Zelenyi, L. M., Milovanov, A. V., and Zimbardo, G. Multiscale magnetic structure of the distant tail: self-consistent fractalapproach, in: New Perspectives on the Earth's magnetotail, eds. Nishida, A., Baker, D. N., and Cowley, S. W. H. 1998. Geophys. Mon. Ser. 105, AGU, 321. 


\section{Figure captions}

Figure 1: The variations of (a) SYM-H index and (b) AE index during the Bastille day event July 13-19, 2000 (marked as the storm phase) and 7 days prior to the event from July 06-12, 2000 (marked as calm phase).

Figure 2. The surrogate data analysis of multifractal parameters for $A E$ index considering the Bastille day geomagnetic storm event and the comparative calm time prior to the event (a) variation of mass exponents with $q$ during disturbance time (b) variation of $f(\alpha)$ versus $\alpha$ during disturbance time (c) variation of generalized Hurst exponent $H(q)$ versus $q$ during disturbance time (d) variation of mass exponents with $q$ during calm time (e) variation of $f(\alpha)$ versus $\alpha$ during calm time (f) variation of generalized Hurst exponent $H(q)$ versus $q$ during calm time. The bold magenta (for storm time) and cyan (for calm time) colored curves represent multifractal parameter of the actual index while mean values of parameters for surrogates are represented as bold blue curves. The corresponding sum and difference of the mean value and the standard deviation is represented as dotted blue curves.

Figure 3. The surrogate data analysis of multifractal parameters for SYM-H index considering the Bastille day geomagnetic storm event and the comparative calm time prior to the event (a) variation of mass exponents with $q$ during disturbance time (b) variation of $f(\alpha)$ versus $\alpha$ during disturbance time (c) variation of generalized Hurst exponent $H(q)$ versus $q$ during disturbance time (d) variation of mass exponents with $q$ during calm time (e) variation of $f(\alpha)$ versus $\alpha$ during calm time (f) variation of generalized Hurst exponent $H(q)$ versus $q$ during calm time. The bold red (for storm time) and green (for calm time) colored curves represent multifractal parameter of the actual index while mean values of parameters for surrogates are represented as bold blue curves. The corresponding sum and difference of the mean value and the standard deviation is represented as dotted blue curves.

Figure 4. Rescaled range analysis of (a) AE index during storm time (b) AE index during calm time (c) SYM-H index during storm time and (d) SYM-H index during calm time. The red line indicates the straight-line fit. Here also the Bastille day geomagnetic storm event and the comparative calm time prior to the event has been used.

Figure 5. The temporal variations of multifractality of (a) AE index during the period of 19902007 (b) SYM-H index during the period of 1985-2007. The values are computed for a moving monthly window of 30 days with an overlap of 23 days.

Figure 6. Sunspot cycle dependence of multifractal strength of AE by considering $\Delta \alpha$ of $\mathrm{AE}$ index and sunspot cycle mode of its IMFn, computed during the period of 1985-2007 (a) Sunspot cycle IMFn of sunspot number (b) $\Delta \alpha$ on a 15 day window (c) Sunspot cycle IMFn of $\Delta \alpha$ on a 15 day window (d) $\Delta \alpha$ on a 30 day window (e) Sunspot cycle IMFn of $\Delta \alpha$ on a 30 day window (f) $\Delta \alpha$ on a 60 day window (g) Sunspot cycle IMFn of $\Delta \alpha$ on a 60 day window. 
Figure 7. Sunspot cycle dependence of multifractal singularity strength $\Delta \alpha$ of AE, SYM-H and Dst indices by considering sunspot cycle (11-year) mode of $\Delta \alpha$ of corresponding indices on 15day, 30-day and 60-day windows (a) AE index (b) SYM-H index and (c) Dst index. The 11-year mode of sunspot number is also provided for comparison.

Figure 8. Sunspot cycle dependence of monofractal Hurst exponent $H_{R / S}$ of AE, SYM-H and Dst indices by considering sunspot cycle (11-year) mode of $H_{R / S}$ of corresponding indices on 15day, 30-day and 60-day windows (a) AE index (b) SYM-H index and (c) Dst index. The 11-year mode of sunspot number is also provided for comparison. 


\section{Tables}

Table 1. The Pearson's correlation coefficients of sunspot cycle mode of SSN and singularity strength of geomagnetic indices during the period of 1985-2007

\begin{tabular}{|c|c|c|c|}
\hline Window span & $\boldsymbol{\Delta} \boldsymbol{\alpha}_{\boldsymbol{A E}}$ & $\boldsymbol{\Delta} \boldsymbol{\alpha}_{\boldsymbol{S Y M}-\boldsymbol{H}}$ & $\boldsymbol{\Delta} \boldsymbol{\alpha}_{\boldsymbol{D} \boldsymbol{s t}}$ \\
\hline 15 day & -0.3263 & 0.7059 & 0.8164 \\
\hline 30 day & -0.4193 & 0.6049 & 0.8808 \\
\hline 60 day & -0.1853 & 0.6534 & 0.8731 \\
\hline
\end{tabular}

Table 2. The Pearson's correlation coefficients of sunspot cycle mode of SSN and $H_{R / S}$ of geomagnetic indices during the period of 1985-2007

\begin{tabular}{|c|c|c|c|}
\hline Window span & $\boldsymbol{H}_{\boldsymbol{A E}}$ & $\boldsymbol{H}_{\boldsymbol{S Y M}-\boldsymbol{H}}$ & $\boldsymbol{H}_{\boldsymbol{D S t}}$ \\
\hline 15 day & 0.5335 & 0.8604 & 0.8540 \\
\hline 30 day & 0.6945 & 0.8568 & 0.9398 \\
\hline 60 day & 0.7248 & 0.7535 & 0.9234 \\
\hline
\end{tabular}


Figures

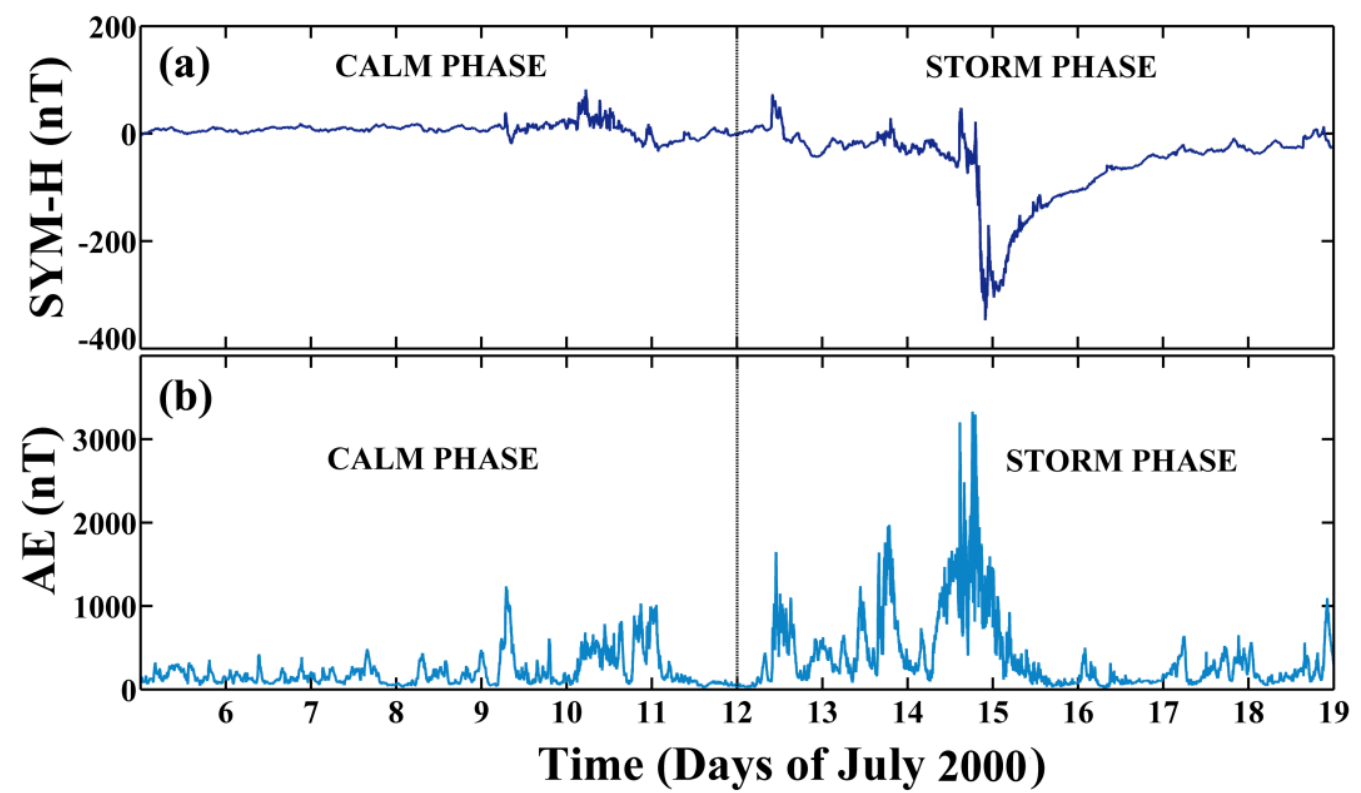

Figure 1: The variations of (a) SYM-H index and (b) AE index during the Bastille day event July 13-19, 2000 (marked as the storm phase) and 7 days prior to the event from July 06-12, 2000 (marked as calm phase). 
AE (DISTURBANCE TIME)
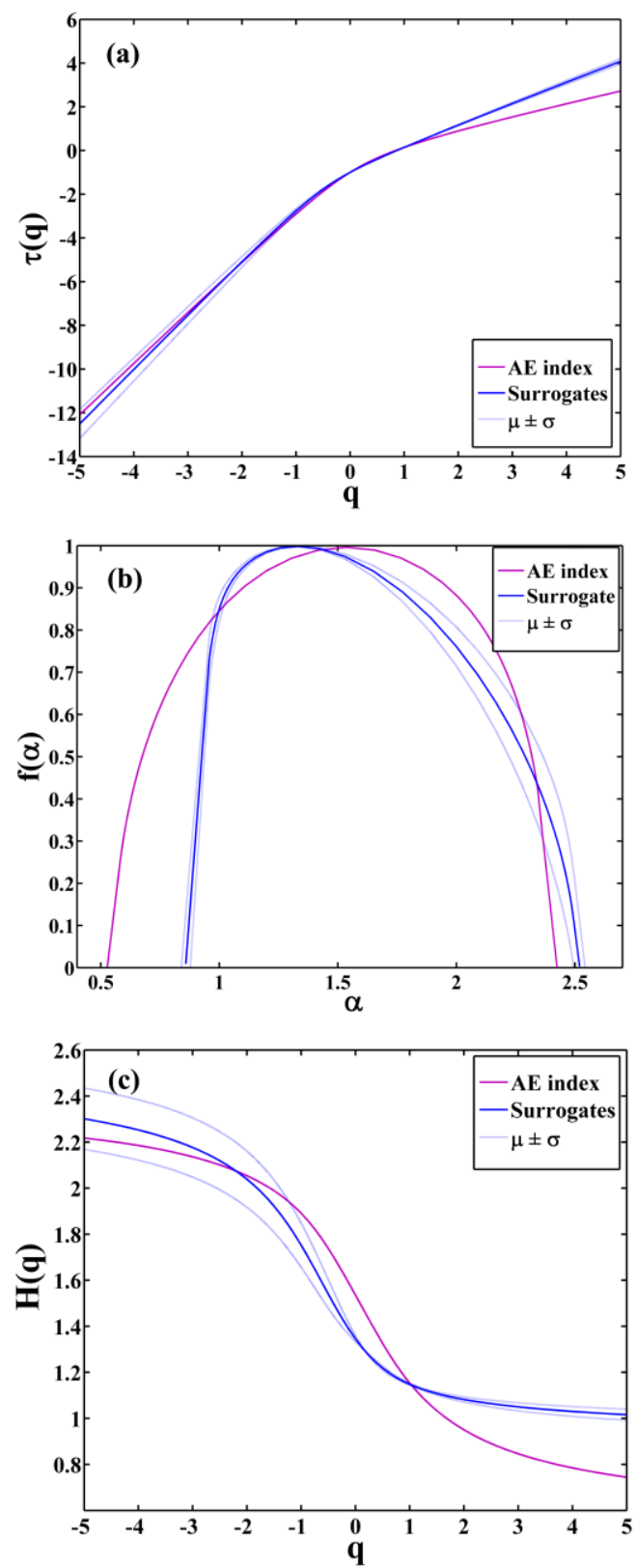

AE (CALM TIME)
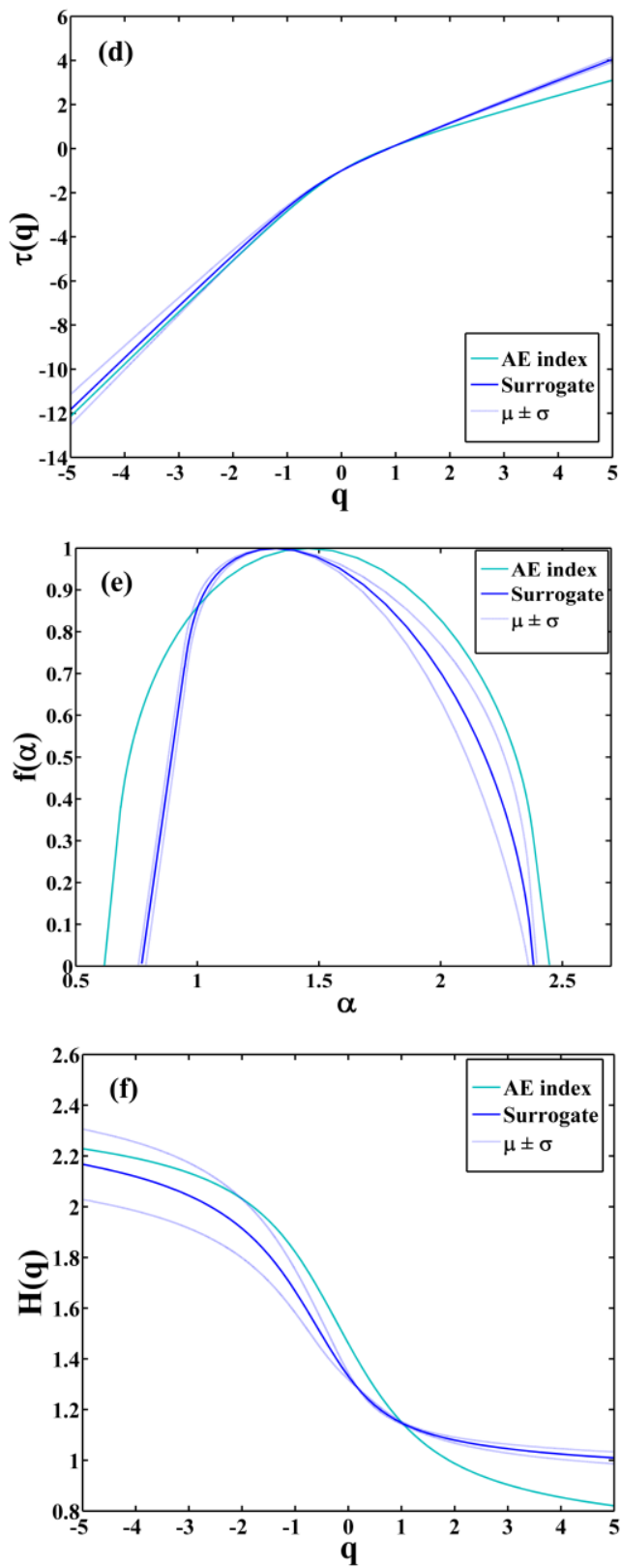

Figure 2. The surrogate data analysis of multifractal parameters for AE index considering the Bastille day geomagnetic storm event and the comparative calm time prior to the event (a) variation of mass exponents with $q$ during disturbance time (b) variation of $f(\alpha)$ versus $\alpha$ during disturbance time (c) variation of generalized Hurst exponent $H(q)$ versus $q$ during disturbance time (d) variation of mass exponents with $q$ during calm time (e) variation of $f(\alpha)$ versus $\alpha$ during calm time (f) variation of generalized Hurst 
exponent $H(q)$ versus $q$ during calm time. The bold magenta (for storm time) and cyan (for calm time) colored curves represent multifractal parameter of the actual index while mean values of parameters for surrogates are represented as bold blue curves. The corresponding sum and difference of the mean value and the standard deviation is represented as dotted blue curves.

SYM-H (DISTURBANCE TIME)
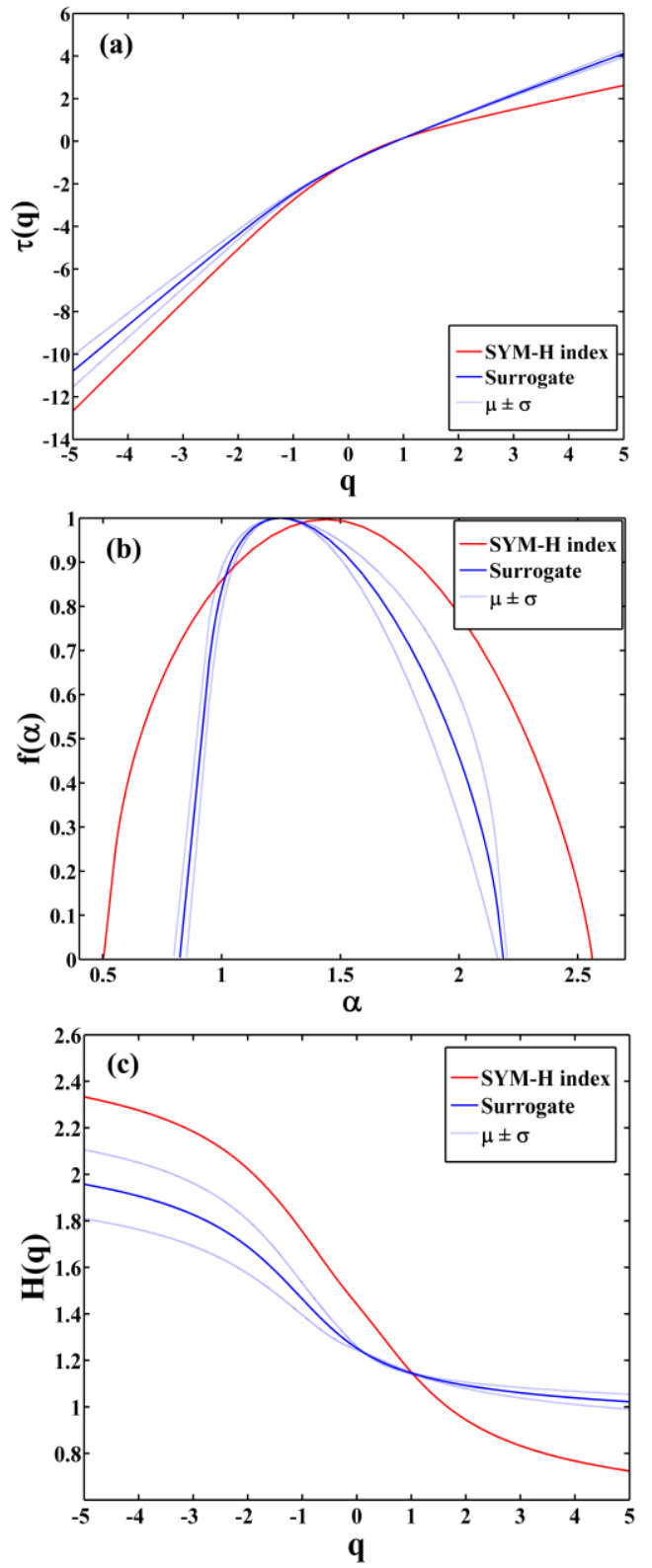

SYM-H (CALM TIME)
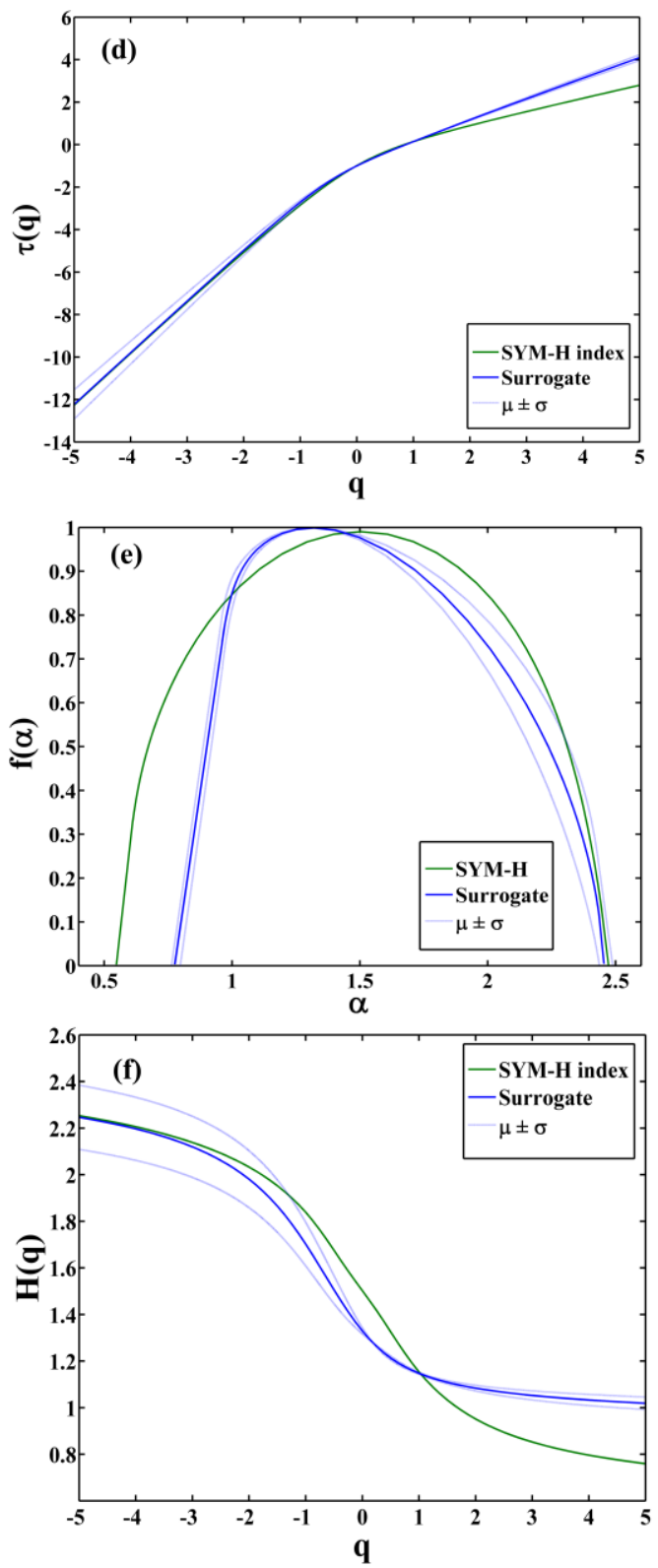
Figure 3. The surrogate data analysis of multifractal parameters for SYM-H index considering the Bastille day geomagnetic storm event and the comparative calm time prior to the event (a) variation of mass exponents with $q$ during disturbance time (b) variation of $f(\alpha)$ versus $\alpha$ during disturbance time (c) variation of generalized Hurst exponent $H(q)$ versus $q$ during disturbance time (d) variation of mass exponents with $q$ during calm time (e) variation of $f(\alpha)$ versus $\alpha$ during calm time (f) variation of generalized Hurst exponent $H(q)$ versus $q$ during calm time. The bold red (for storm time) and green (for calm time) colored curves represent multifractal parameter of the actual index while mean values of parameters for surrogates are represented as bold blue curves. The corresponding sum and difference of the mean value and the standard deviation is represented as dotted blue curves.
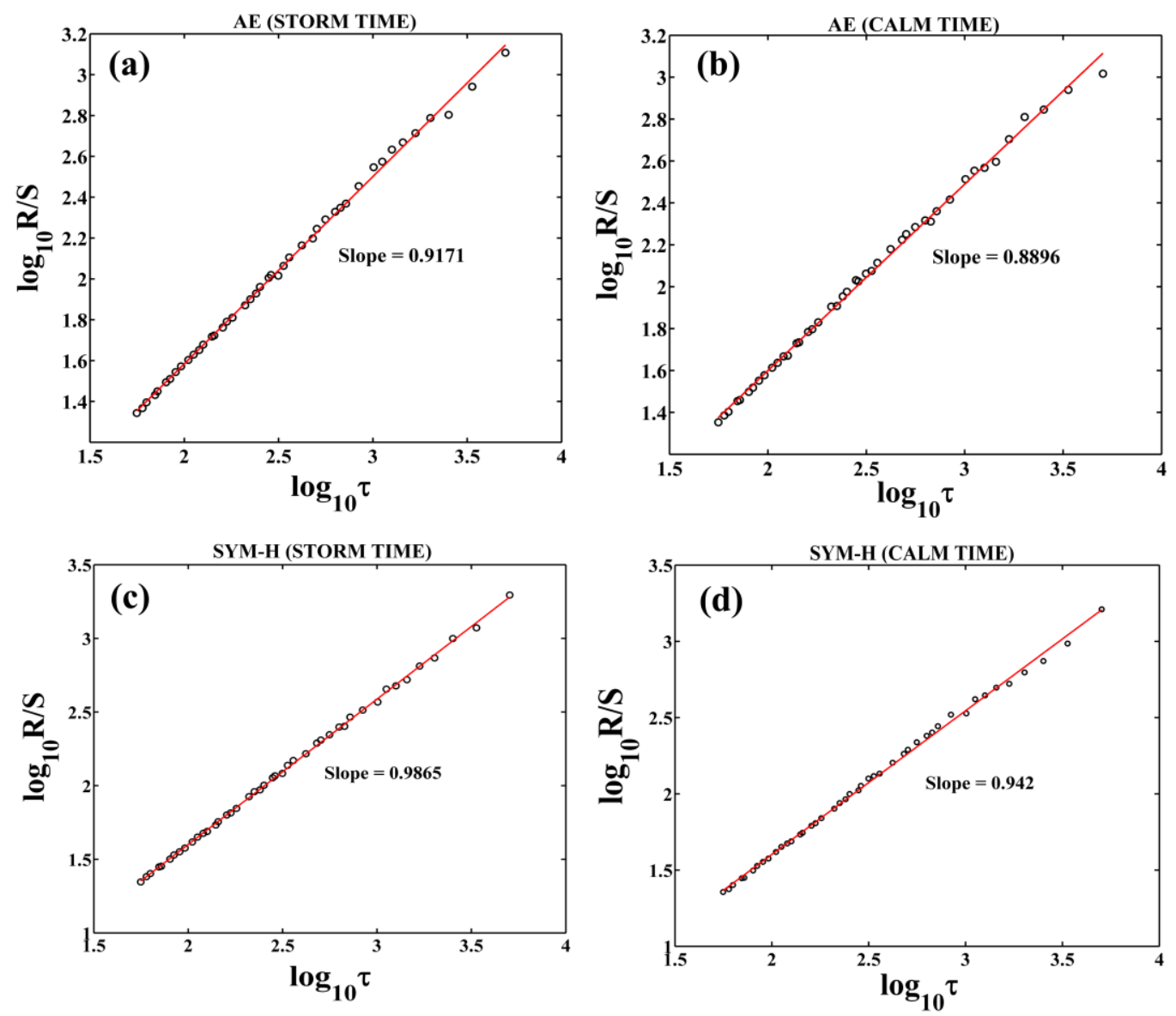

Figure 4. Rescaled range analysis of (a) AE index during storm time (b) AE index during calm time (c) SYM-H index during storm time and (d) SYM-H index during calm time. 
The red line indicates the straight-line fit. Here also the Bastille day geomagnetic storm event and the comparative calm time prior to the event has been used.
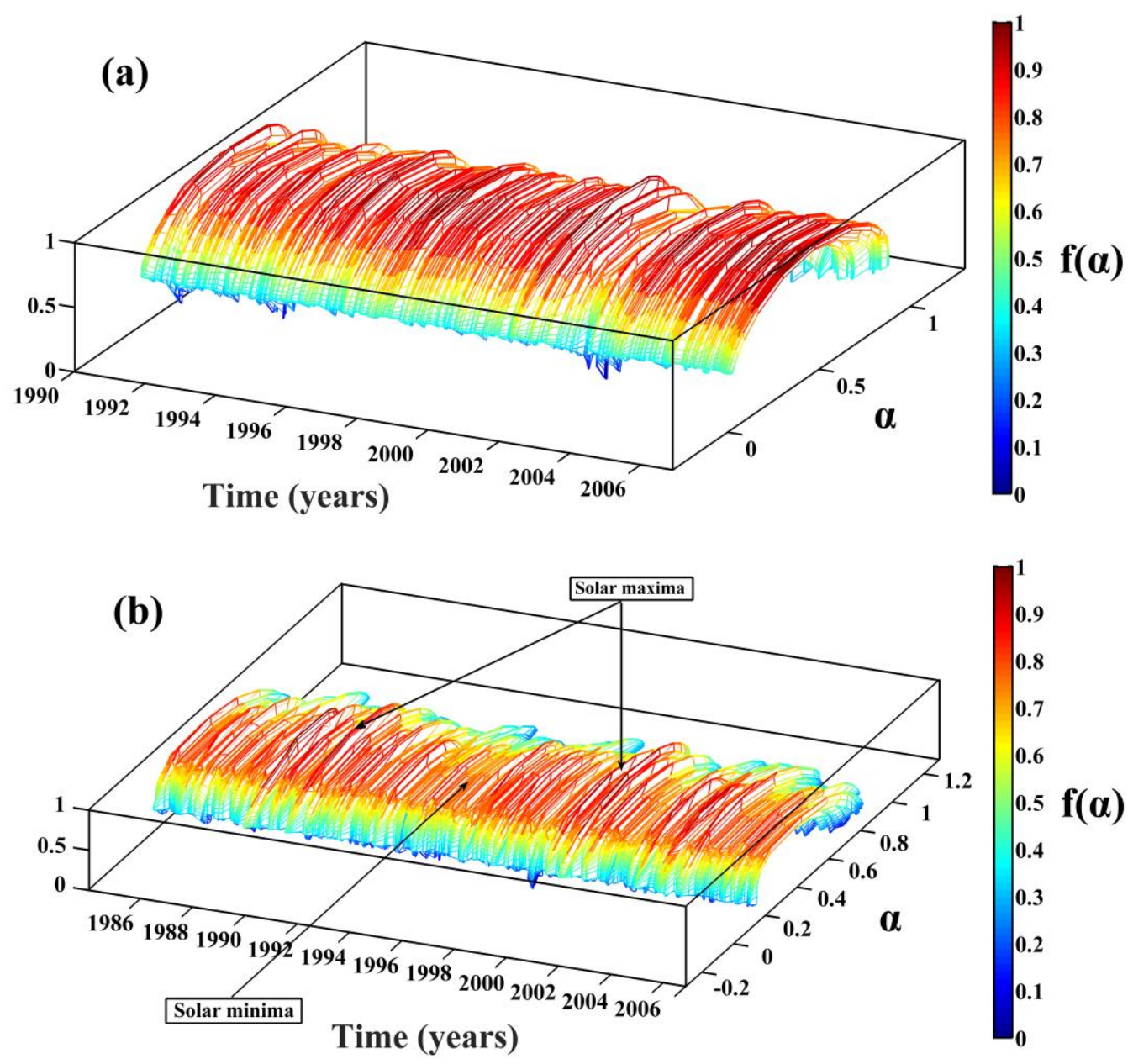

Figure 5. The temporal variations of multifractality of (a) AE index during the period of 1990-2007 (b) SYM-H index during the period of 1985-2007. The values are computed for a moving monthly window of 30 days with an overlap of 23 days. 
Sunspot cycle dependence of $\Delta \alpha_{\mathrm{AE}}$
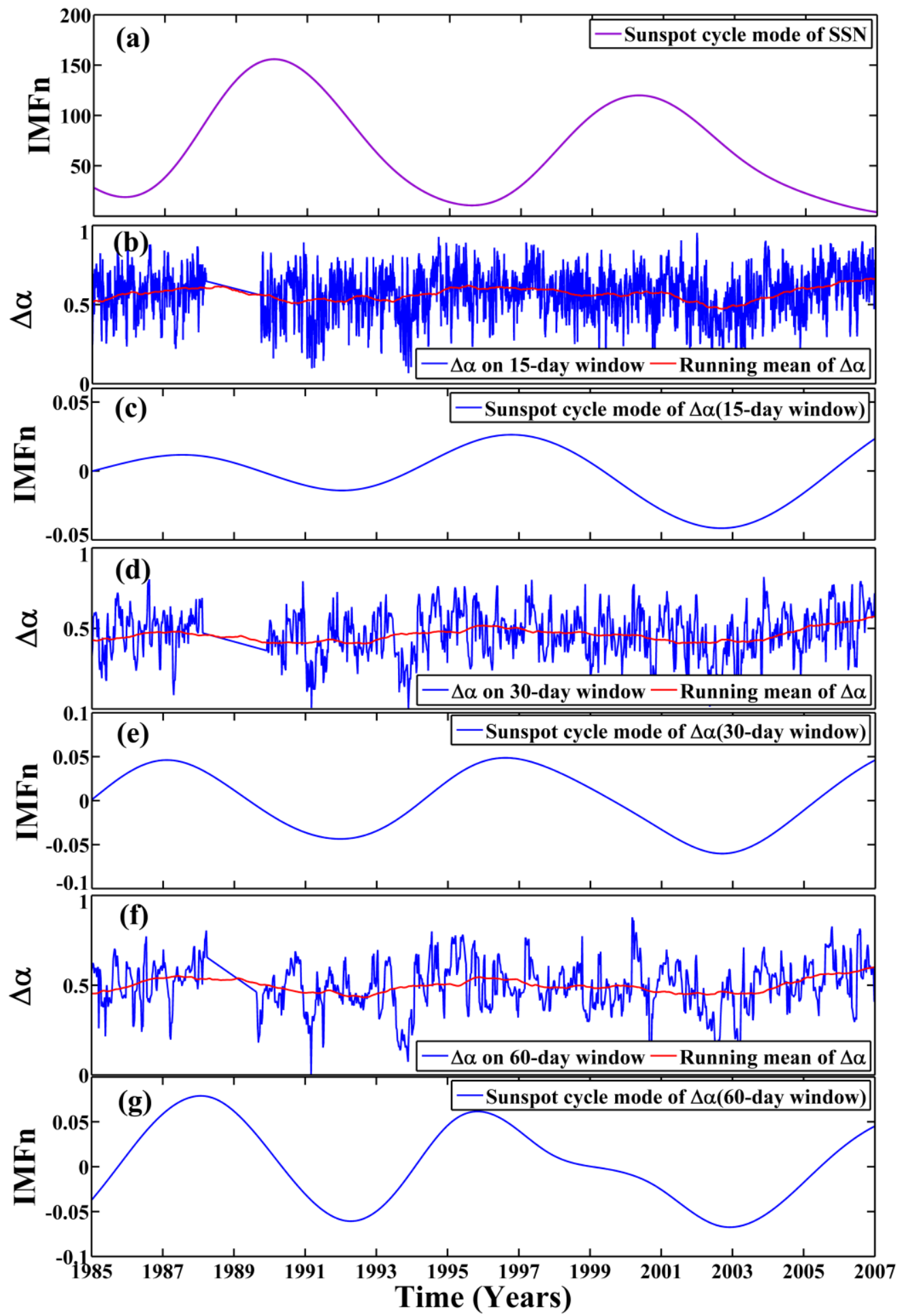

6 
Figure 6. Sunspot cycle dependence of multifractal strength of AE by considering $\Delta \alpha$ of AE index and sunspot cycle mode of its IMFn, computed during the period of 1981-2011 (a) Sunspot cycle IMFn of sunspot number (b) $\Delta \alpha$ on a 15 day window (c) Sunspot cycle IMFn of $\Delta \alpha$ on a 15 day window (d) $\Delta \alpha$ on a 30 day window (e) Sunspot cycle IMFn of $\Delta \alpha$ on a 30 day window (f) $\Delta \alpha$ on a 60 day window (g) Sunspot cycle IMFn of $\Delta \alpha$ on a 60 day window. 


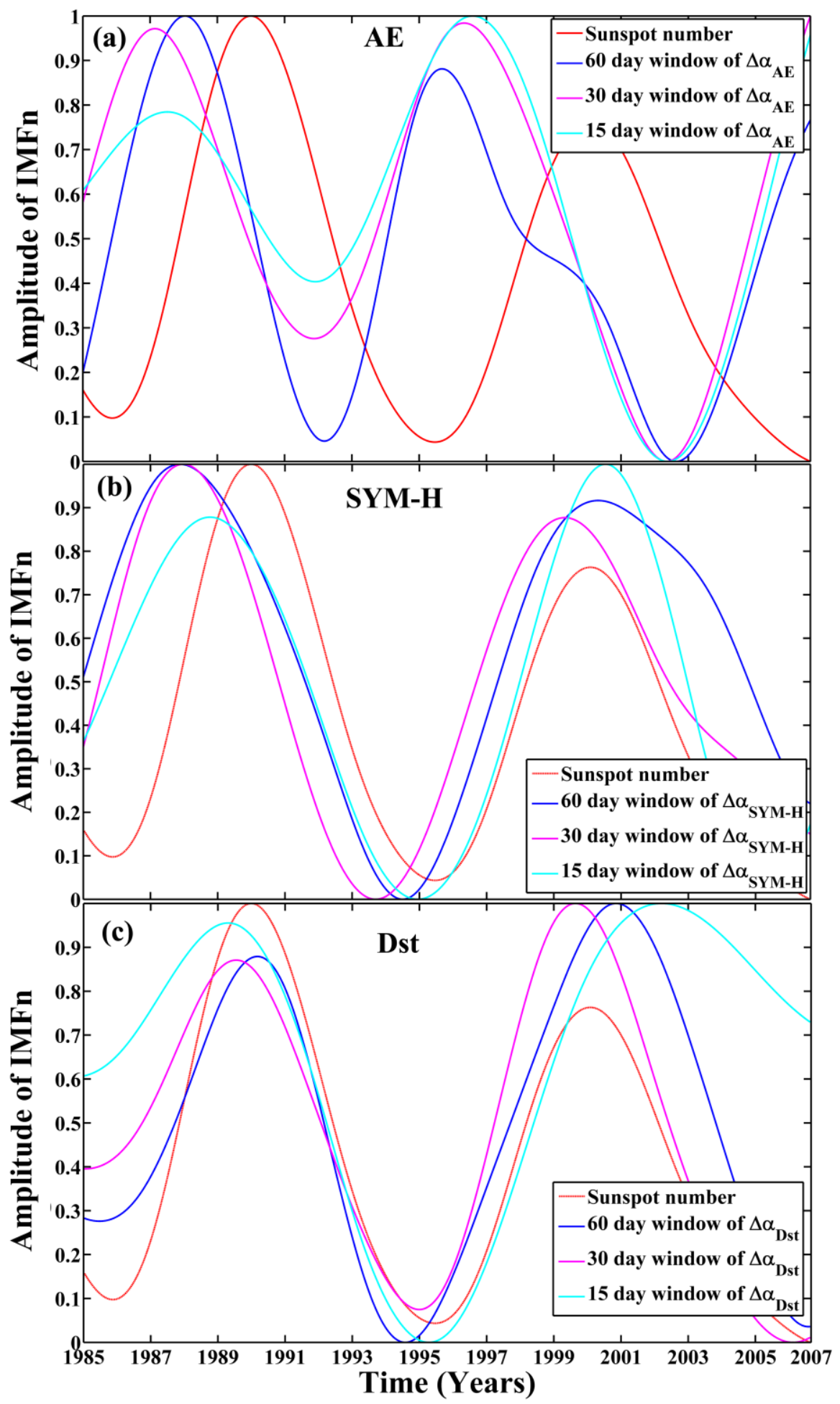


Figure 7. Sunspot cycle dependence of multifractal singularity strength $\Delta \alpha$ of AE, SYM$\mathrm{H}$ and Dst indices by considering sunspot cycle (11-year) mode of $\Delta \alpha$ of corresponding indices on 15-day, 30-day and 60-day windows (a) AE index (b) SYM-H index and (c) Dst index. The 11-year mode of sunspot number is also provided for comparison. 

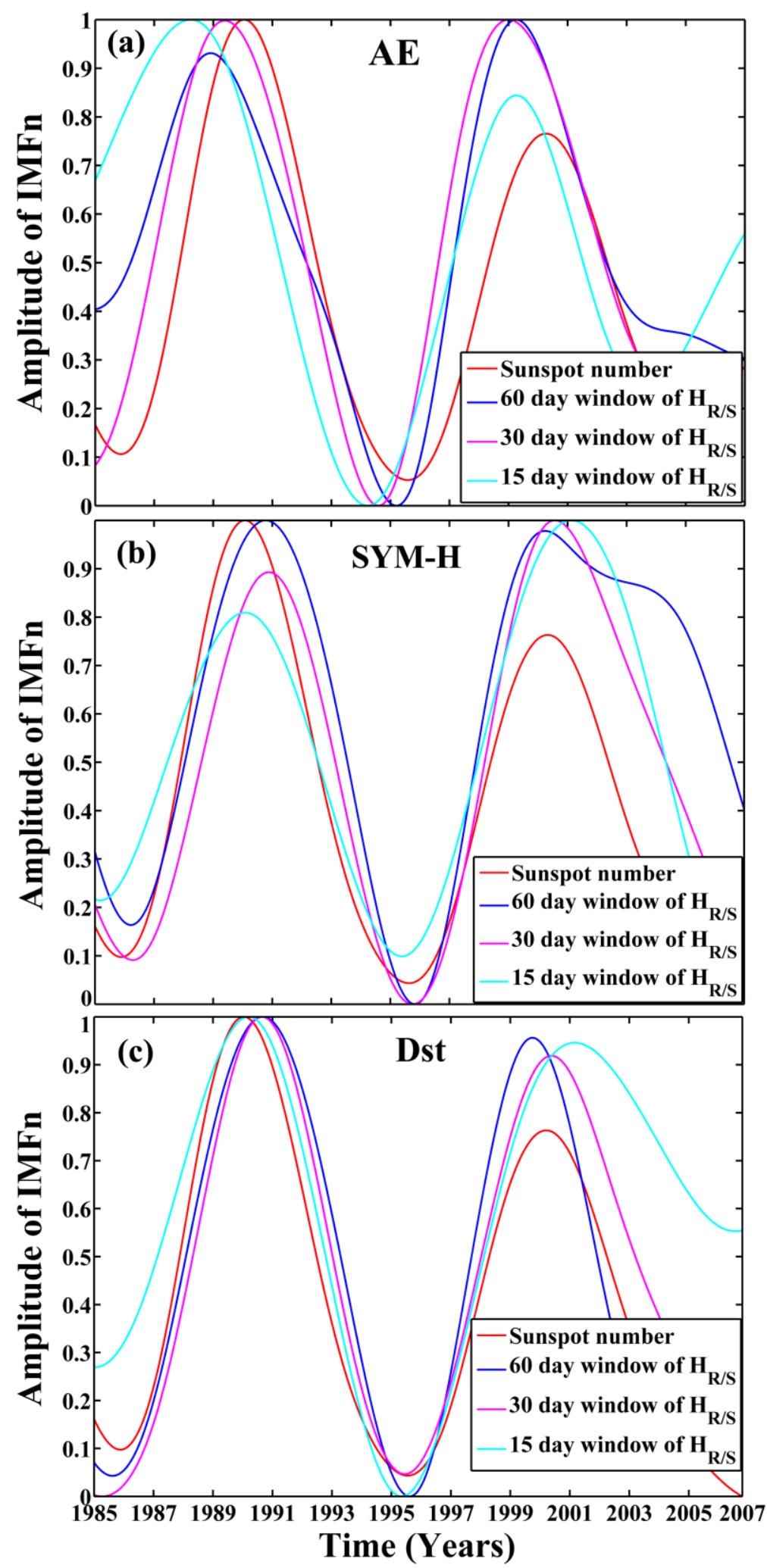